\title{
Anabases
}

ANABASES Traditions et réceptions de l'Antiquité

$4 \mid 2006$

Varia

\section{Le Daremberg ou l'Antiquité en images}

\section{François Lissarrague}

\section{OpenEdition}

Journals

Édition électronique

URL : http://journals.openedition.org/anabases/2971

DOI : 10.4000/anabases.2971

ISSN : 2256-9421

\section{Éditeur}

E.R.A.S.M.E.

\section{Édition imprimée}

Date de publication : 1 octobre 2006

Pagination : 173-177

ISSN : 1774-4296

\section{Référence électronique}

François Lissarrague, "Le Daremberg ou l'Antiquité en images », Anabases [En ligne], 4| 2006, mis en ligne le 01 janvier 2012, consulté le 21 octobre 2019. URL : http://journals.openedition.org/anabases/ 2971 ; DOI : 10.4000/anabases.2971 
Anabases 4 (2006), p. 173-177

\section{Le Daremberg ou l'Antiquité en images}

FRANÇOIS LISSARRAGUE

La MISE EN LIGNE du $D A$ est une excellente nouvelle, dont on ne peut que se réjouir, et dont il faut féliciter ceux qui en ont pris l'initiative tout comme ceux qui ont mené l'entreprise à terme. Mais j'aimerais aussitôt ajouter qu'on ne peut en rester là : l'objet de cette journée est précisément de réfléchir à ce qui nous paraît souhaitable, possible, indispensable.

Pour ma part, avant d'entrer dans le détail de ce qui me vient à l'esprit, j'aimerais souligner quelques aspects de l'histoire du $D A$, du point de vue du statut donné à l'image dans l'érudition antiquaire, car cette histoire conditionne le mode de production des données iconographiques et leur usage.

Publié de 1877 à 1919 (ce sont les dates imprimées sur les pages de titre des 10 volumes du $D A$ ), le projet, comme nous le rappelle C. Valenti, remonte à 1850, et le premier contrat date de $1855^{1}$. La chronologie peut être affinée, si l'on tient compte de la publication par fascicules, et il faudra mieux dater les articles mis en ligne ${ }^{2}$, afin d'en relativiser d'emblée l'actualité. L'usage des documents figurés dans un dictionnaire

1 Contrat publié par D. GouREvich, "Un épisode de l'histoire du DA..." ", in Les Archéologues et l'archéologie, Colloque de Bourg-en-Bresse, Caesarodunum, XXVII, 1993, p. 79-81.

2 Voir la thèse de Ph. RouET, Attribuer, classer. Sur l'historiographie moderne des vases attiques XVIII -XXe s., soutenue à Paris 1 en 1996 et publiée par l'Atelier de Lille. Le travail porte sur la comparaison entre J. Beazley et E. Pottier ; on y trouvera, p. 520-548, une bio-bibliographie d'E. Pottier, et la date de tous ses articles du $D A$, par fascicules. La version anglaise de cette thèse, Approaches to the Study of Greek Vases. Beazley and Pottier, Oxford 2001, ne contient malheureusement pas ces éléments. 
d'antiquités, tels qu'il se déploie dans le $D A$, n'est pas nouveau. On peut le faire remonter au grand ouvrage du bénédictin Dom Bernard de Montfaucon, L'Antiquité expliquée et représentée en figures, 5 tomes en 10 volumes, suivis d'un supplément en cinq volumes, publiés en 1719 et 1724 . Entreprise colossale, faite de gravures tirées d'un immense trésor de dessins, un musée de papier, offrant au lecteur 1120 planches gravées, et près de 40000 figures, mises en ordre, non pas alphabétiquement, mais selon un plan thématique inspiré de la classification des antiquitates de Varron. Monfaucon, dans sa préface de 1719 , précise :

Il s'agit ici de toute l'Antiquité : on en rapporte toutes les parties, on donne sur chacune un grand nombre de figures : ces figures sont expliquées avec toute l'exactitude et toute la précision dont j'ai été capable. [...] Je réduis dans un corps d'ouvrage toute l'Antiquité : par ce terme d'Antiquité j'entends seulement tout ce qui peut tomber sous les yeux et ce qui se peut représenter dans les images.

Le succès de l'entreprise permet l'édition d'un Supplément en 1724, dans la préface duquel Montfaucon ajoute :

Les images tirées des monuments sont presque les effets d'une descente sur les lieux et nous mettent devant les yeux ce que nous n'entendions qu'à demi. [...] Les images nous instruisent plus sûrement sur bien des choses que les auteurs eux-mêmes, et augmentent beaucoup le plaisir de la lecture. Un autre avantage non moins considérable qu'on peut tirer de ces monuments c'est la connaissance d'un grand nombre de choses que les auteurs n’apprennent pas.

Ainsi sont nettement affirmés plusieurs présupposés qui circulent implicitement ou explicitement dans la culture archéologique du XVIII et du XIXe siècles. Les images sont plus vraies que les textes, elles expliquent mieux que les mots, elles sont immédiates, d'une évidence directe. Elles produisent sur le lecteur non seulement un certain plaisir, mais un puissant effet de réel.

Salomon Reinach, dans la nécrologie qu'il consacre à E. Saglio, rapporte un des ses propos: " Je n'aime pas qu'on renvoie à Montfaucon ${ }^{3}$ ». Il avait raison de condamner un ouvrage vieux de 150 ans (1724/1877) ; et nous aurions tort de remettre en circulation, tel quel, un ouvrage vieux de 130 ans (1877/2006). Pourtant l'écart entre Montfaucon et Saglio n'est peut-être pas aussi grand que celui qui nous sépare aujourd'hui de Saglio.

En tout cas, du point de vue des usages de l'image, le $D A$ ne differe pas, dans son principe, de L'Antiquité expliquée de Montfaucon. Il utilise l'image comme un document direct, un moyen explicatif ; les 7608 figures du $D A$ ont pour fonction de préci- 
ser - sur le mode documentaire - l'exposé que contient chaque article. E. Saglio le souligne dans sa préface ${ }^{4}$ :

Les gravures, qui sont nombreuses, sont toutes puisées aux sources antiques, soit que les dessins aient été faits d'après les monuments, soit qu'ils aient été pris dans des ouvrages qui les reproduisent fidèlement. [...] Il suffira d'un regard jeté sur ce livre pour s'apercevoir que les gravures n'y sont pas de pures illustrations, mais des preuves à l'appui de ce qui est allégué dans le texte,

et il poursuit en citant Winckelmann :

Selon moi ce sont les images qui doivent décider du sens des passages des livres anciens qui, exposant des choses connues dans ces temps-là, ne sont jamais aussi claires qu'il le faudrait pour bien les entendre dans les siècles où les usages et les mœurs ont totalement changé.

Saglio ne s'écarte pas des principes édictés cent ans plus tôt par le fondateur de l'archéologie "moderne ». L'évidence visuelle est au service de l'intelligence des textes, de la philologie qui rabat la documentation figurée sur l'écrit. L'image rend explicite tout ce qui relève de la vie matérielle, tout ce qui dans les textes, reste souvent implicite.

Ces principes sont énoncés sur le même mode quelques années avant le $D A$, dans un dictionnaire de taille bien plus modeste, mais qui connut un large succès et qui n'est probablement pas étranger à la décision des éditions Hachette, conseillées par Daremberg, de se lancer dans l'entreprise du DA. Il s'agit du petit Dictionnaire des Antiquités romaines et grecques, rédigé par un voyageur anglais, A. Rich. L'édition originale, publiée à Londres en 1849 porte un titre révélateur : Illustrated companion to the Latin dictionnary and Greek lexikon : forming a glossary of all the words representing visible objects connected with the arts and every-day life of the Greeks and Romans. L'édition française, parue chez Firmin Didot en 1859, connut une large diffusion, prouvant l'utilité d'un tel type d'ouvrage. Accompagné de 2000 gravures, pour un texte in $8^{\circ}$ de 715 p., il fait une très large place à l'image et, privilégiant une logique lexicale, les entrées suivent l'ordre alphabétique des mots latins. On a déjà là l'organisation du $D A$. La préface d'A. Rich insiste longuement sur le rôle du dessin, et sur les qualités nécessaires à la gravure : fidélité au monument original, exactitude dans la reproduction, minutie dans le rendu du détail. L'auteur insiste :

Il n'est guère nécessaire de s'étendre sur l'avantage d'employer la représentation des œuvres d'art comme secours pour interpréter les textes. [...] Chaque dessin est ici un commentaire pratique du sens des mots, adressé à l'esprit par la vue, et non pas une gravure d'agrément, destinée à embellir une page d'impression. 
L'image est donc un outil, le vecteur d'une clarté que revendique à son tour Saglio, pour le $D A$. Ce faisant, il crée un stock d'images qui sera largement exploité par les éditions Hachette, dans toute une série d'œuvres dérivées parmi lesquelles on peut signaler :

Victor Duruy, Histoire des Grecs. Nouvelle édition revue et augmentée, enrichie d'environ 2000 gravures, 3 vol., Paris, Hachette 1887-89 (la première édition date de 1851);

G. Fougères, La vie publique et privée des Grecs et des Romains : album contenant plus de 890 gravures, Paris, Hachette 1894 ;

Plus directement la version abrégée du $D A$, par Pierre Lavedan, Dictionnaire illustré de la mythologie et des arts grecs et romains, avec 1013 figures, Paris, Hachette 1931;

enfin, le Gaffiot, dont le titre exact est Dictionnaire illustré Latin-Français paru en 1934, et sans cesse réédité, (jusqu'à sa récente refonte, en 2000, qui, sous le titre Le grand Gaffiot, en a expurgé les images). La préface de F. Gaffiot précisait :

$8^{\circ}$ L'idée d'illustrer l'ouvrage et sa réalisation reviennent aux Editeurs ; mon rôle s'est borné à émettre des avis.

Cette idée éditoriale, puissamment déclinée à travers ces nombreuses publications, a créé en France une mémoire visuelle quasi inconsciente pour des générations de latinistes ; et elle a sans doute pesé lourd dans notre perception de l'Antiquité.

Aujourd'hui, avec un $D A$ en ligne, nous retrouvons ce matériel, remis à disposition des chercheurs et des curieux. Parce que la technique le permet, ces images sont aisément exportables, et c'est un beau cadeau qui nous est fait là ; parce que le livre est quasi centenaire, il est libre de droits et c'est un service rendu à tous les enseignants. On peut donc imaginer sans difficulté qu'il en sera fait un large usage, par les temps qui courent, où la politique de rentabilité des biens culturels impose des droits souvent exorbitants sur les images. Et le risque est bien là, par économie, par facilité, de reconduire un état largement dépassé du savoir iconographique.

Depuis des années en effet, nous avons été nombreux à remettre en cause l'usage immédiat de l'image, à analyser l'écart entre le réel et sa représentation, à poser la question du statut des images dans la culture qui les produit. Nombreux aussi à souligner qu'il n'y a pas d'image indépendamment du support qui la véhicule, et qu'un relief, un vase ou une monnaie n'ont ni la même fonction, ni le même impact visuel ; ils n'entrent pas dans les mêmes dispositifs, ne circulent pas de la même façon et sont perçus différemment.

L'urgence est donc, de ce point de vue, de compléter les indications matérielles, muséographiques, chronologiques qui permettront de mieux situer chaque figure du $D A$, de replacer le détail dans l'ensemble auquel il appartient, de situer chaque objet dans la série dont il relève et de donner les outils bibliographiques modernes qui permettent d'en faire un usage critique. Il faut aussi dépasser le dessin au trait qui clari- 
fie, certes, mais souvent reproduit ce que le dessinateur comprend et n'est pas exempt d'erreurs ${ }^{5}$, renvoyer aux bases de données modernes qui fournissent des photographies, des vues variées permettant de confronter des aspects divers, des cadrages différents.

Il me semble qu'il y a là un vaste chantier qui s'ouvre, auquel pour ma part je suis prêt à participer en ce qui concerne la céramique grecque ${ }^{6} .7608$ figures : de quoi s'occuper, seul ou en équipe, pour un bon moment.

FRANÇOIS LISSARRAGUE

Centre Louis Gernet

2 rue Vivienne

75002 Paris

flissa@ehess.fr

5 Sur ce point, voir les remarques de Carl Robert, dans son Archaeologische Hermeneutik, Berlin 1919, trad. italienne, Ermeneutica archeologica, Naples 1976, chapitre 1 : «Voir, dessiner, décrire ".

6 On trouvera une proposition de commentaire à la figure $618 \mathrm{du} D A$. Une telle entreprise entre dans d'autres programmes analogues du Centre Gernet, sur l'histoire de la céramique grecque. Elle prolongerait utilement la base mise en route par M. Denoyelle "Connaissance des vases Grecs " qui porte sur les publications de vases des XVIII" et XIX siècles. 